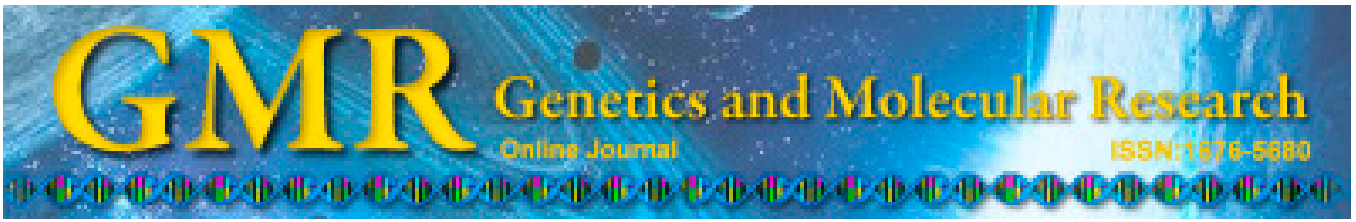

\title{
Disease indicators for sepsis and analysis of sepsis treatment in children using the continuous blood purification technique
}

\author{
J.P. Liu, X.W. Wang and L.P. Qie \\ Pediatric Department of Hematology, Inner Mongolia People’s Hospital, \\ Huhhot, China \\ Corresponding author: J.P. Liu \\ E-mail: liujianpingdh@yeah.net
}

Genet. Mol. Res. 14 (2): 5685-5693 (2015)

Received September 17, 2014

Accepted December 3, 2014

Published May 25, 2015

DOI http://dx.doi.org/10.4238/2015.May.25.21

\begin{abstract}
We analyzed disease severity, inflammation markers, and dynamic changes in cartilage glycoprotein 39 (YKL-40) and Creactive protein (CRP) levels in children with sepsis before and after treatment with continuous blood purification (CBP). Study participants were 30 children with severe sepsis who were cured from the disease (experimental group) in the Children's Serious Disease Center of Inner Mongolia People's Hospital between June 2012 and October 2013. Symptomatic CBP treatment was performed after disease severity scoring. Interleukin-6 (IL-6), tumor necrosis factor- $\alpha$ (TNF- $\alpha$ ), YKL-40, and CRP levels were tested $0,12,24$, and $48 \mathrm{~h}$ after CBP treatment. YKL-40 mRNA expression in whole blood was determined biochemically, and its expression in peripheral blood was determined with an immunochemical method. We found a significant difference in disease severity scores before and $48 \mathrm{~h}$ after CBP treatment $(\mathrm{P}<0.05)$. IL6 , TNF- $\alpha$, YKL-40, and CRP levels in children with sepsis at 12, 24, and $48 \mathrm{~h}$ after CBP treatment significantly differed from those before treatment $(\mathrm{P}<0.05)$. The relative expression of YKL-40 mRNA in the
\end{abstract}


experimental group before CBP treatment significantly increased from that of the control group ( $\mathrm{P}<0.05)$. We found a positive correlation between IL-6, TNF- $\alpha$, YKL-40, and CRP levels $48 \mathrm{~h}$ after CBP treatment. In conclusion, CBP is an effective treatment strategy for pediatric sepsis. YKL-40 and CRP can be used to evaluate the effects of sepsis treatment.

Key words: Continuous blood purification technique; Sepsis; Children; Treatment mechanism; Cartilage glycoprotein 39; C-reactive protein

\section{INTRODUCTION}

Sepsis is a systemic inflammatory response syndrome (SIRS), which is the number one cause of intensive care unit deaths and a serious problem in adult and pediatric critical care medicine (Ishimaru et al., 2013). In recent years, morbidity associated with sepsis has been rising annually. Sepsis is the number one cause of death among critically ill children (Caribe et al., 2013). In recent years, several studies on active interventions for sepsis using continuous blood purification (CBP) have been reported in China and internationally. CBP has been shown to prevent and treat severe sepsis (Peng et al., 2010). During treatment of sepsis, CBP lowers the overall in vivo peak concentration of inflammatory mediators, down-regulates the body's inflammatory response. Thus, CBP does not only target a single inflammatory maker, but reduces the effect of sepsis on both endothelial cells and hemodynamics (Rimmele and Kellum, 2011). CBP also maintains the responsiveness of cells to endotoxemia and bacteremia and promotes recovery of the patient's immunity (Nacionales et al., 2012). Although CBP has been widely used and studied, the mechanism of CBP treatment is unclear. No study on the changes in protein markers before and after CBP treatment of children with sepsis has been conducted.

We have therefore compared the clinical effects of CBP treatment on children with sepsis. The dynamic changes in the protein markers cartilage glycoprotein 39 (YKL-40, or also chitinase-3-1ike-1) and C-reactive protein (CRP) were analyzed. We discuss the indications and opportunities for CBP treatment of sepsis and its potential mechanism.

\section{MATERIAL AND METHODS}

\section{Study participants}

Thirty children who met the diagnostic criteria for severe sepsis and were cured in the Children's Serious Disease Center at Inner Mongolia People's Hospital from June 2012 to October 2013 were included in our study. The control group consisted of 30 healthy children who were examined in the outpatient clinic during the same period.

The details of the experimental and control groups were as follows.

Experimental group: aged 60 days to 12 years; 4 newborns, weighing $\geq 3.6 \mathrm{~kg} ; 14$ children weighing $<10 \mathrm{~kg}$ and $16>10 \mathrm{~kg}$; disease severity score upon admission: 21 children, $<70$ points and $9,70-80$ points. The severity of the children diagnosed as having sepsis upon admission was scored $<80$ points, including those with an SIRS stage above Grade 3 [including refractory shock, multiple organ dysfunction syndrome (MODS)] and CBP treatment administered (Xiong et al, 2009). 
Control group: aged 60 days to 12 years; 3 newborns, weighing $\geq 3.4 \mathrm{~kg}$; 12 children weighing $<10 \mathrm{~kg}$ and 18 weighing $>10 \mathrm{~kg}$.

No significant differences in age and body weight were found between the experimental and control groups.

The diagnostic criteria for severe sepsis were based on the diagnostic criteria for pediatric sepsis released by the International Sepsis Conference, SAN Antonio, 2005. Severe sepsis is accompanied by one of the following conditions: cardiovascular dysfunction, respiratory distress syndrome, or dysfunction of 2 or more other organs (Thompson et al., 2013).

\section{Conventional and CBP treatment}

Conventional comprehensive treatment, treatment of primary diseases, antibiotic treatment, and nutritional symptomatic and supportive treatment were administered to all 30 children before CBP treatment to maintain the acid-base equilibrium and water-electrolyte balance. All children required mechanical ventilation for assisted respiration, and 17 children required dopamine and noradrenaline to maintain blood pressure.

CBP treatment was administered to the children who did not exhibit signs of significant recovery following the conventional comprehensive treatment and who met the diagnostic criteria for severe sepsis. Intubation with a double-lumen tube was performed through the femoral or jugular vein with a Model BM25 CBP machine (Baxter, Deerfield, FL, USA) and child-type tubing. All children were subjected to continuous venous blood filtration. The in vitro circulation line (blood line + filter) was pre-filled with plasma, and whole blood filling. For anticoagulation, heparin was administered to children with normal coagulation function, and low molecular heparin was administered to children with slightly abnormal post-operative coagulation function. The preclinical improved displacement liquid was provided at the hospital bedside in accordance with the acid-base equilibrium and electrolyte conditions of the children. The details of the solutions were as follows: Solution A: normal saline $(3000-3250 \mathrm{~mL}), 5 \%$ glucose $(250-500 \mathrm{~mL})$, water for injection $(500-750 \mathrm{~mL})$, calcium chloride $(1-1.5 \mathrm{~g}), 25 \%$ magnesium sulfate $(3 \mathrm{~mL})$, and $10 \%$ potassium chloride $(0-12 \mathrm{~mL})$; Solution B: $5 \%$ sodium bicarbonate (250-375 mL). Solution A and Solution B entered the line simultaneously. Back replacement (3-4 h) was followed by front replacement and was performed for all diseased children. The total replacement volume in $24 \mathrm{~h}$ was $1-1.5 \mathrm{~L} / \mathrm{kg}$. Slow ultrafiltration was performed while plasma or albumin was transfused at the beginning of CBP treatment. The ultrafiltration speed was increased after blood pressure was normal. CBP treatment was performed for $24-48 \mathrm{~h}$ initially and for $12 \mathrm{~h}$ daily or $24 \mathrm{~h}$ every other day thereafter, for 3-12 days in total (Ikumi et al., 2013).

\section{Severity scoring}

In May 1995, the emergency treatment team of the Academy of Pediatrics of the Chinese Medical Association discussed and passed the Scoring Method for Pediatric Critical Cases. This publication defines 10 measurement indexes, including those for heart rate, blood pressure, respiration, partial pressure of blood oxygen, $\mathrm{pH}$, volume ratio of erythrocytes, gastrointestinal related manifestations, and serum sodium, serum potassium, creatinine, or urea nitrogen levels. Considering relevant international scoring methods and national conditions, pediatric diseases were classified as extremely critical, critical, and non-critical based on these scores (Piquereau et al., 2013). 


\section{Specimen determination}

Determination of biochemical indexes was performed using VS2 automatic biochemical analyzer (Abaxis, Union City, CA, USA), and blood gas analysis was conducted using ABL90 FLEX blood-gas analyzer (Radiometer, Copenhagen, Denmark). Cytokine levels [interleukin-6 (IL-6) and tumor necrosis factor- $\alpha(\mathrm{TNF}-\alpha)$ ] were determined with the enzymelinked immunosorbent assay (ELISA) method, and serum YKL-40 levels were determined in accordance with the instructions of the ELISA kit (Xitang Biotechnology Co., Ltd., Shanghai, China). The reagent for CRP determination was provided by Kang Pu Hui Wei Technology Co., Ltd. (Beijing, China) and serum CRP levels were determined using immunoturbidimetry.

\section{Relative expression of YKL-40 mRNA in whole blood}

Whole blood was drawn from the children, and $1 \mathrm{~mL}$ TRIZOL was added to obtain a TRIZOL homogenate. Chloroform $(0.2 \mathrm{~mL})$ was added to each TRIZOL homogenate. The sample was vortexed for $30 \mathrm{~s}$, then placed on the bench for $15 \mathrm{~min}$, and finally centrifuged at $12,000 \mathrm{~g}$ at $4^{\circ} \mathrm{C}$ for $15 \mathrm{~min}$. Isopropanol $(0.5 \mathrm{~mL})$ was added to the supernatant. The sample was then mixed, placed at room temperature for $10 \mathrm{~min}$, and centrifuged at $4^{\circ} \mathrm{C}$ at $12,000 \mathrm{~g}$ for $10 \mathrm{~min}$. The supernatant was discarded, and $75 \%$ ethyl alcohol $(0.8 \mathrm{~mL})$ was added. After centrifugation for $10 \mathrm{~min}$ at $8000 \mathrm{~g}$ at $4^{\circ} \mathrm{C}$, the sample was mixed gently and alcohol washed twice. The ethyl alcohol was discarded, and the EP tube was placed in a fume hood to dry the remaining ethyl alcohol. Rnase/Dnase water $(20 \mu \mathrm{L})$ was added to the pellet, and the solution was pipetted several times. The solution $(10 \mu \mathrm{L})$ was added to $590 \mu \mathrm{L}$ sterile water. Cary UV4000 (Agilent, Santa Clara, CA, USA) was used to determine the purity and concentration of the RNA. The Primescript ${ }^{\mathrm{TM}}$ RT reagent (Sigma, USA) was used to perform inverse transcription to obtain cDNA, and RNA concentration was determined (based on the instructions).

\section{Detection of YKL-40 in peripheral blood using an immunochemical method}

Venous blood was drawn, and heparin was used for anticoagulation. The above liquid was diluted with phosphate buffered saline (PBS) in a ratio of 1:1, mixed, and centrifuged (2000 rpm, $20 \mathrm{~min}$ ) after the volume reached the liquid level of the glucan-meglumine diatrizoate. The peripheral blood mononuclear cells (PBMCs) were centrifugationed (1500 rpm for $10 \mathrm{~min}$ ) after being pipetted, and their concentration was adjusted to $1 \times 10^{6} / \mathrm{L}$ using PBS. The PBMCs were cultured at a concentration of $1 \times 10^{6} / \mathrm{L}$ and digested with $0.25 \%$ pancreatinethylenediaminetetraacetic acid (EDTA) after the cells achieved a stable growth status and exhibited logarithmic growth. The cell solution ( $1 \mathrm{~mL} /$ well) was dropped onto a polylysinecoated slide after the culture medium had been adjusted to a concentration of $1 \times 10^{4} / \mathrm{mL}$. After incubation ( $48 \mathrm{~h}$ ), the slides were fixed with $4 \%$ paraformaldehyde for $15 \mathrm{~min}$, treated with $0.1 \%$ TritonX-100, and blocked in goat serum for $10 \mathrm{~min}$. The excess blocking solution was discarded, and YKL-40 antibody (1:500) was added. The samples were stored at $4{ }^{\circ} \mathrm{C}$ overnight. They were then incubated with the ready-to-use goat anti-rabbit secondary antibody (Abcam, Cambridge, UK) for $20 \mathrm{~min}$ and the rabbit anti-human third antibody for 20 min. The slides were washed thrice with PBS for 5 min, developed in 3,3'-diaminobenzidine (DAB), dehydrated in gradient alcohol, clarified in dimethylbenzene solution, sealed with neutral gum, and microscopically observed for YKL-40 protein expression. 


\section{Statistical analysis}

The SPSS 13.0 (IBM, Chicago, IL, USA) software was used for statistical analysis of the experimental data. All data are reported as means \pm standard deviation (SD). Pairwise comparisons were performed at various time points with the least significant difference (LSD) method. A $t$-test of two independent samples was conducted to compare IL- 6 and TNF- $\alpha$ levels of the experimental group with those of the control group $48 \mathrm{~h}$ after CBP treatment. A Satterthwaite approximate $t$-test was used in case of heterogeneity of variance. $\mathrm{P}<0.05$ indicated a significant difference. A Pearson correlation analysis of YKL-40, CRP, TNF- $\alpha$, and IL-6 levels was conducted for disease severity scoring before and after CBP treatment of children with sepsis.

\section{RESULTS}

\section{Disease severity scores before and after CBP treatment of children with sepsis}

Severity score is $57.15 \pm 14.62$ at $0 \mathrm{~h}$, and $83.25 \pm 17.48$ at $48 \mathrm{~h}$. We found a significant difference in the disease severity scores before and $48 \mathrm{~h}$ after CBP treatment $(\mathrm{P}=0.004)$.

\section{Variation in inflammatory marker (IL-6 and TNF- $\alpha$ ) levels}

We compared IL-6 and TNF- $\alpha$ levels at 12, 24, and $48 \mathrm{~h}$ after CBP treatment in children with sepsis with those before treatment and found a significant difference $(\mathrm{P}<0.05)$. IL-6 and TNF- $\alpha$ levels exhibited a trend for a decrease after treatment. We also compared serum IL-6 and TNF- $\alpha$ levels between the experimental group (children with sepsis) and the control group, and our results showed a statistically significant difference $(\mathrm{P}<0.05$; Tables 1 and 2$)$.

\begin{tabular}{|c|c|c|c|c|c|}
\hline Inflammatory marker & $0 \mathrm{~h}$ & $12 \mathrm{~h}$ & $24 \mathrm{~h}$ & $48 \mathrm{~h}$ & P value \\
\hline IL-6 (ng/L) & $695.2 \pm 168.3$ & $407.7 \pm 115.2 *$ & $241.5 \pm 82.4^{*}$ & $197.4 \pm 76.4^{*}$ & $<0.001$ \\
\hline TNF $-\alpha(n g / L)$ & $1074.5 \pm 285.4$ & $736.1 \pm 254.5^{*}$ & $527.4 \pm 189.7 *$ & $394.5 \pm 148.7 *$ & $<0.001$ \\
\hline
\end{tabular}

$\mathrm{CBP}=$ continuous blood purification; IL- $6=$ interleukin- 6 ; TNF- $\alpha=$ tumor necrosis factor alpha. $* \mathrm{P}<0.001$.

Table 2. Comparison of IL- 6 and TNF- $\alpha$ levels between the experimental group (children with sepsis) and the control group $48 \mathrm{~h}$ after CBP treatment.

\begin{tabular}{lccc}
\hline Inflammatory marker & Control group & Experimental group (children with sepsis) & P value \\
\hline $\mathrm{IL}-6(\mathrm{ng} / \mathrm{L})$ & $87.2 \pm 28.5$ & $197.4 \pm 76.4^{*}$ & $<0.001$ \\
$\mathrm{TNF}-\alpha(\mathrm{ng} / \mathrm{L})$ & $117.4 \pm 42.1$ & $394.5 \pm 148.7^{*}$ & $<0.001$ \\
\hline
\end{tabular}

For abbreviations, see Table 1. $* \mathrm{P}<0.001$.

\section{Changes in marker protein YKL-40 and CRP concentrations}

YKL-40 and CRP protein levels at 12, 24, and $48 \mathrm{~h}$ after CBP treatment of children with sepsis were compared with those before treatment, revealing a statistically significant difference $(\mathrm{P}<0.05)$. The concentration of YKL-40 in the peripheral blood of children with 
sepsis $48 \mathrm{~h}$ after CBP treatment was compared to that in healthy children, and no significant difference was found (Tables 3 and 4).

Table 3. YKL-40 and CRP protein concentration in children with sepsis before and after CBP treatment.

\begin{tabular}{|c|c|c|c|c|c|}
\hline Index & $0 \mathrm{~h}$ & $12 \mathrm{~h}$ & $24 \mathrm{~h}$ & $48 \mathrm{~h}$ & $P$ value \\
\hline YKL-40 $(\mu \mathrm{g} / \mathrm{L})$ & $214.1 \pm 68.3$ & $152.4 \pm 44.7$ & $108.3 \pm 62.5$ & $62.7 \pm 22.8$ & $<0.001$ \\
\hline $\mathrm{CRP}(\mu \mathrm{g} / \mathrm{L})$ & $589.4 \pm 182.4$ & $472.7 \pm 138.6$ & $336.5 \pm 108.1$ & $146.5 \pm 87.2$ & $<0.001$ \\
\hline
\end{tabular}

$\mathrm{CBP}=$ continuous blood purification; YKL-40 = cartilage glycoprotein $39 ; \mathrm{CRP}=\mathrm{C}$-reactive protein.

Table 4. Comparison of YKL-40 and CRP concentrations between the experimental group (children with sepsis) and the control group $48 \mathrm{~h}$ after CBP treatment.

\begin{tabular}{lccr}
\hline Index & Control group & Experimental group (children with sepsis) & P value \\
\hline YKL-40 $(\mu \mathrm{g} / \mathrm{L})$ & $51.2 \pm 21.4$ & $62.7 \pm 22.8$ & 0.056 \\
CRP $(\mu \mathrm{g} / \mathrm{L})$ & $76.6 \pm 29.5$ & $146.5 \pm 87.2$ & $<0.001$ \\
\hline
\end{tabular}

For abbreviations, see Table 3.

\section{Analysis of the relative expression of YKL-40 mRNA}

Comparison of the relative expression of YKL- 40 mRNA at different time points after CBP treatment with that before treatment revealed no significant difference $(\mathrm{P}>0.05)$. When YKL-40 expression before CBP treatment was compared between the experimental and control groups, we found a significant difference $(\mathrm{P}<0.05)$, but no difference was found for YKL-40 levels after CBP treatment $(\mathrm{P}>0.05)$. The relative expression of YKL-40 mRNA $\left(2^{-\triangle \Delta C t}\right)$ in the peripheral blood of the children with sepsis was lower $0,12,24$, and $48 \mathrm{~h}$ after CBP treatment than the relative expression before treatment (Tables 5 and 6).

Table 5. Variations in the relative expression of YKL- 40 mRNA in children with sepsis before and after CBP treatment.

\begin{tabular}{|c|c|c|c|c|c|}
\hline Index & $0 \mathrm{~h}$ & $12 \mathrm{~h}$ & $24 \mathrm{~h}$ & $48 \mathrm{~h}$ & $P$ value \\
\hline$\Delta \mathrm{Ct}$ & $4.25 \pm 1.12$ & $5.31 \pm 1.26$ & $6.04 \pm 1.33$ & $5.57 \pm 1.28$ & 0.183 \\
\hline $2^{-\Delta \Delta \mathrm{Ct}}$ & $1(0.5-1.5)$ & $0.6(0.3-0.9)$ & $0.6(0.4-0.8)$ & $0.5(0.2-0.8)$ & \\
\hline
\end{tabular}

$\mathrm{CBP}=$ continuous blood purification; YKL-40 = cartilage glycoprotein $39 ; \mathrm{Ct}=$ cycle threshold.

Table 6. Relative expression of YKL- $40 \mathrm{mRNA}$ in the experimental group (children with sepsis) compared to that in the control group before and $48 \mathrm{~h}$ after CBP treatment.

\begin{tabular}{lccc}
\hline Index & Control group & $0 \mathrm{~h}$ & $48 \mathrm{~h}$ \\
\hline$\Delta \mathrm{Ct}$ & $6.33 \pm 1.31$ & $4.25 \pm 1.12^{*}$ & $5.57 \pm 1.28$ \\
$\mathrm{P}$ value & & 0.033 & 0.272 \\
\hline
\end{tabular}

For abbreviations, see Table 5. $* \mathrm{P}<0.05$.

\section{YKL-40 expression in peripheral blood using the cellular immunochemical method}

YKL-40 was expressed in mononuclear cells of peripheral blood cells (Figure 1). 


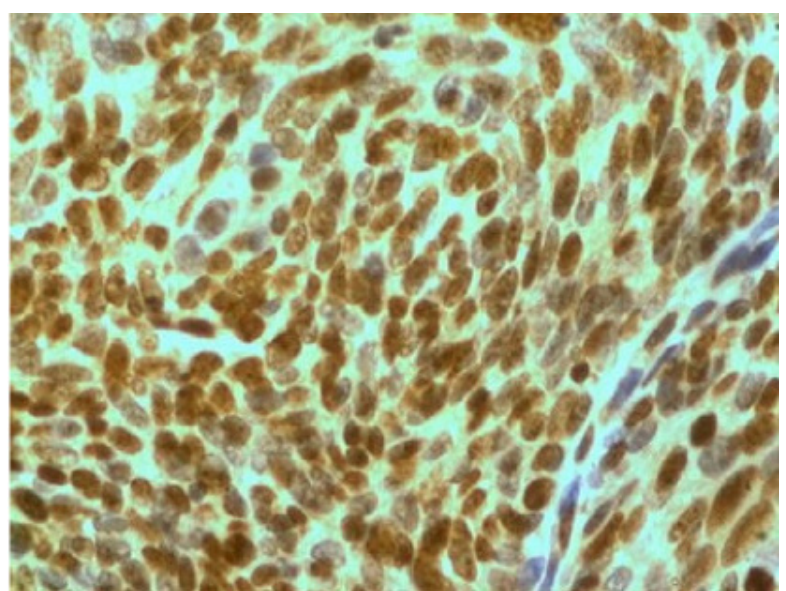

Figure 1. Localization of YKL-40 expression in children with sepsis. Brown color indicates a positive reaction.

\section{Correlation between YKL-40 and CRP and IL-6 and TNF- $\alpha$ concentrations}

The concentrations of YKL-40 and IL-6, YKL-40 and TNF- $\alpha$, CRP and IL- 6 , and CRP and TNF- $\alpha$ all exhibited a significant positive correlation $(r=0.832,0.634,0.893$, and 0.727 , respectively; $\mathrm{P}<0.05$; Table 7).

Table 7. Correlation between YKL-40 and CRP and IL-6 and TNF- $\alpha$ concentrations in peripheral blood in children with sepsis before and after CBP treatment.

\begin{tabular}{lcc}
\hline Correlation coefficient $(\mathrm{r})$ & YKL-40 & CRP \\
\hline IL-6 & $0.832^{*}$ & $0.893^{*}$ \\
TNF- $\alpha$ & $0.634^{*}$ & $0.727^{*}$ \\
\hline
\end{tabular}

$\mathrm{CBP}=$ continuous blood purification; $\mathrm{CRP}=\mathrm{C}$-reactive protein; IL-6 = interleukin-6; TNF- $\alpha=$ tumor necrosis factor- $\alpha$; YKL-40 = cartilage glycoprotein $39 . * \mathrm{P}<0.05$.

\section{DISCUSSION}

Sepsis is associated with serious infections, and exacerbated SIRS may cause multiple organ failure. Bacterial infections are one of the major causes of sepsis (Vanmassenhove et al., 2013). The interaction between the host and the microorganism activates the natural immunity pathway and triggers defensive mechanisms in the host, including body fluid composition and cells. Mononuclear cells release the pro-inflammatory markers and cytokines IL-1, IL-6, and TNF- $\alpha$ (Betue and Deutz, 2013; Qin et al., 2013). Sepsis is a pathological process involving the excessive cells and humoral immunity caused by infection with bacteria and toxins. A large number of soluble inflammatory factors are involved in MODS (Lai et al., 2013).

CBP uses a continuous operation method to increase the blood flow volume during extracorporeal circulation (Chancharoenthana et al., 2013). The use of filters with a high permeability and good biocompatibility, transfusion of a large amount of displacement liquid, and equipment with a highly accurate fluid balance system provide this methodology with the following advantages: stable hemodynamics, continuous and stable control of azotemia 
and the water-electrolyte metabolism, continuous elimination of toxins and intermediate molecular weight substances in the blood stream, provision of nutritional supplements and drug treatment on demand, and achievement of a stable balance in critical patients (Saxena et al., 2012; Strateva et al., 2012). CBP can be used in patients with low blood pressure and can also create nutrition support.

YKL-40 is a carbohydrate-binding protein of the chitinase family that does not have chitinase activity, and has a molecular weight of $40 \mathrm{kDa}$. Its crystal structure is known, but its biological functions remain unclear (Kastelijn et al., 2013; Kornblit et al., 2013). YKL-40 may play a role in acute and chronic inflammatory processes. Existing studies have demonstrated that it is a growth factor found in connective tissue cells and can promote the migration of endothelial cells as well as the degradation and re-formation of the extracellular matrix (Tatar et al., 2013). YKL-40 is secreted by different types of inflammatory cells. In vivo studies have shown that YKL-40 is secreted by activated macrophages and neutrophil granulocytes in patients with bacterial infection (Tuten et al., 2014).

$\mathrm{CRP}$ is an acute phase protein. CRP concentration has a higher predictive value than white blood cell count and neutrocyte count in children with concealed bacteremia. The predictive value has a reference value based on critically ill children with unknown disease causes (Abdollahi et al., 2012). TNF- $\alpha$ and IL-6 levels are associated with inflammation, and the concentrations of TNF- $\alpha$ and IL- 6 in infection pediatric patients rise. A continuous concentration of IL-6 of $>500 \mathrm{pg} / \mathrm{mL}$ is associated with a prolonged course of abdominal sepsis and an increase in morbidity in children (Celik et al., 2010). Our data demonstrate that YKL-40 and CRP are both reactants during the acute stage of sepsis. YKL-40 levels can thus predict the therapeutic effect of CBP and prognosis in children with sepsis.

Our findings have great clinical significance. Most importantly, we have revealed the optimal time to start CBP therapy - immediately after the diagnosis of severe sepsis in children. Research by Soeorg et al. (2013) indicated that CBP timing is crucial, and the therapeutic effect of CBP is decreased when dysfunctions occur in 5-6 visceral organs. After fluid resuscitation, sick children are not able to maintain their fluid balance through their own urination mechanism. This results in an enlarged liver, and increased or emerging moist rales in the lung. Alternatively, CBP treatment should be performed when the fluid overload accounts for over $10 \%$ of the body weight (Baraboutis et al., 2010). CBP treatment of children with sepsis can significantly improve their oxygenation capacity, treat acidosis, stabilize blood pressure, and remove medium molecular weight substances and inflammatory molecules. It has also been shown to significantly improve the recovery rate of children with sepsis (House and Ronco, 2008).

Production and release of YKL-40 may be stimulated by inflammatory cytokines (Knudsen et al., 2009). IL-6 and TNF- $\alpha$ are two important inflammatory markers that play important roles during sepsis (Nielsen et al., 2011). Studies have shown that TNF- $\alpha$ and IL-6 levels are associated with inflammation, and that their levels rise in patients with sepsis. In our study, IL-6 and TNF- $\alpha$ levels in children with sepsis started to decrease significantly $12 \mathrm{~h}$ after CBP treatment (showing the same trend for a decrease as YKL-40). We show that the IL-6 and TNF- $\alpha$ concentrations had a positive correlation with the YKL-40 concentration, indicating that the increase in IL-6 can activate production of YKL-40.

\section{REFERENCES}

Abdollahi A, Shoar S, Nayyeri F and Shariat M (2012). Diagnostic value of simultaneous measurement of procalcitonin, interleukin-6 and hs-CRP in prediction of early-onset neonatal sepsis. Mediterr. J. Hematol. Infect. Dis. 4: e2012028. 
Baraboutis IG, Doris K, Papanikolaou K, Tsagalou EP, et al. (2010). An outbreak of hemodialysis catheter-related bacteremia with sepsis caused by Streptococcus agalactiae in a hemodialysis unit. Int. J. Infect. Dis. 14: e418-422.

Betue CT and Deutz NE (2013). Changes in arginine metabolism during sepsis and critical illness in children. Nestle Nutr. Inst. Workshop Ser. 77: 17-28.

Caribe RA, Chaves GR, Pocognoni JD and Souza IA (2013). Potential drug interactions in patients with sepsis admitted to the intensive care unit. Farm Hosp. 37: 383-387.

Celik IH, Demirel FG, Uras N, Oguz SS, et al. (2010). What are the cut-off levels for IL-6 and CRP in neonatal sepsis? J. Clin. Lab. Anal. 24: 407-412.

Chancharoenthana W, Tiranathanagul K, Srisawat N, Susantitaphong P, et al. (2013). Enhanced vascular endothelial growth factor and inflammatory cytokine removal with online hemodiafiltration over high-flux hemodialysis in sepsis-related acute kidney injury patients. Ther. Apher. Dial. 17: 557-563.

House AA and Ronco C (2008). Extracorporeal blood purification in sepsis and sepsis-related acute kidney injury. Blood Purif. 26: 30-35.

Ikumi N, Matsukawa Y, Kuwana Y, Kodaira H, et al. (2013). Staphylococcus aureus sepsis after etanercept induction in a hemodialysis patient. Hemodial. Int. 17: 133-135.

Ishimaru N, Ohnishi H, Nishiuma T, Doukuni R, et al. (2013). Antithyroid drug-induced agranulocytosis complicated by pneumococcal sepsis and upper airway obstruction. Intern. Med. 52: 2355-2359.

Kastelijn EA, Moorsel CH, Ruven HJ, Korthagen NM, et al. (2013). YKL-40 and matrix metalloproteinases as potential biomarkers of inflammation and fibrosis in the development of bronchiolitis obliterans syndrome. Sarcoidosis Vasc. Diffuse Lung Dis. 30: 28-35.

Knudsen LS, Hetland ML, Johansen JS, Skjødt H, et al. (2009). Changes in plasma IL-6, plasma VEGF and serum YKL40 during treatment with etanercept and methotrexate or ptanercept alone in patients with active rheumatoid arthritis despite methotrexate therapy. Biomark. Insights 4: 91-95.

Kornblit B, Hellemann D, Munthe-Fog L, Bonde J, et al. (2013). Plasma YKL-40 and CHI3L1 in systemic inflammation and sepsis-experience from two prospective cohorts. Immunobiology 218: 1227-1234.

Lai TS, Wang CY, Pan SC, Huang TM, et al. (2013). Risk of developing severe sepsis after acute kidney injury: a population-based cohort study. Crit. Care 17: R231.

Nacionales DC, Cuenca AG, Ungaro L, Gentile LF, et al. (2012). The acute immunological response to blood transfusion is influenced by polymicrobial sepsis. Shock 38: 598-606.

Nielsen AR, Plomgaard P, Krabbe KS, Johansen JS, et al. (2011). IL-6, but not TNF-alpha, increases plasma YKL-40 in human subjects. Cytokine 55: 152-155.

Peng Z, Singbartl K, Simon P, Rimmelé T, et al. (2010). Blood purification in sepsis: a new paradigm. Contrib. Nephrol. 165: 322-328.

Piquereau J, Godin R, Deschênes S, Bessi VL, et al. (2013). Protective role of PARK2/Parkin in sepsis-induced cardiac contractile and mitochondrial dysfunction. Autophagy 9: 1837-1851

Qin W, Zhang J, Lv W, Wang X, et al. (2013). Effect of carbon monoxide-releasing molecules II-liberated CO on suppressing inflammatory response in sepsis by interfering with nuclear factor kappa B activation. PLoS One 8: e75840.

Rimmele T and Kellum JA (2011). Clinical review: blood purification for sepsis. Crit. Care 15: 205.

Saxena AK, Panhotra BR, Al-hafiz A, Sundaram DS, et al. (2012). Cefotaxime-heparin lock prophylaxis against hemodialysis catheter-related sepsis among Staphylococcus aureus nasal carriers. Saudi J. Kidney Dis. Transpl. 23: 743-754.

Soeorg H, Huik K, Parm U, Ilmoja ML, et al. (2013). Genetic relatedness of coagulase-negative Staphylococci from gastrointestinal tract and blood of preterm neonates with late-onset sepsis. Pediatr. Infect. Dis. J. 32: 389-393.

Strateva T, Kostyanev T and Setchanova L (2012). Ralstonia pickettii sepsis in a hemodialysis patient from Bulgaria. Braz. J. Infect. Dis. 16: 400-401.

Tatar E, Gungor O, Celtik A, Sisman AR, et al. (2013). Correlation between serum YKL-40 (Chitinase-3-like protein 1) level and proteinuria in renal transplant recipients. Ann. Transplant. 18: 95-100.

Thompson JP, Serrano-Gomez A, McDonald J, Ladak N, et al. (2013). The nociceptin/orphanin FQ system is modulated in patients admitted to ICU with sepsis and after cardiopulmonary bypass. PLoS One 8: e76682.

Tuten A, Kucur M, Imamoglu M, Oncul M, et al. (2014). Serum YKL-40 levels are altered in endometriosis. Gynecol. Endocrinol. 30: 381-384.

Vanmassenhove J, Glorieux G, Hoste E, Dhondt A, et al. (2013). Urinary output and fractional excretion of sodium and urea as indicators of transient versus intrinsic acute kidney injury during early sepsis. Crit. Care 17: R234.

Xiong J, Zhu S, Zhou Y, Wu H, et al. (2009). Regulation of omega-3 fish oil emulsion on the SIRS during the initial stage of severe acute pancreatitis. J. Huazhong Univ. Sci. Technolog. Med. Sci. 29: 35-38. 